\title{
Capacitive field-effect (bio-)chemical sensors based on nanocrystalline diamond films
}

\author{
M. Bäcker ${ }^{1,2}$, A. Poghossian ${ }^{1,2}$, M. H. Abouzar ${ }^{1,2}$, S. Wenmackers ${ }^{3}$, S. D. Janssens ${ }^{3}$, K. \\ Haenen $^{3,4}$, P. Wagner ${ }^{3,4}$, and M. J. Schöning ${ }^{1,2}$ \\ ${ }^{1}$ Institute of Nano- and Biotechnologies, Aachen University of Applied Sciences, Campus Jülich, \\ Germany \\ ${ }^{2}$ Institute of Bio- and Nanosystems, Research Centre Jülich, Germany \\ ${ }^{3}$ Institute for Materials Research, Hasselt University, Diepenbeek, Belgium \\ ${ }^{4}$ Division IMOMEC, IMEC vzw., Diepenbeek, Belgium
}

\begin{abstract}
Capacitive field-effect electrolyte-diamond-insulator-semiconductor (EDIS) structures with Oterminated nanocrystalline diamond (NCD) as sensitive gate material have been realized and investigated for the detection of $\mathrm{pH}$, penicillin concentration, and layer-by-layer adsorption of polyelectrolytes. The surface oxidizing procedure of NCD thin films as well as the seeding and NCD growth process on a $\mathrm{Si}_{-} \mathrm{SiO}_{2}$ substrate have been improved to provide high $\mathrm{pH}$-sensitive, non-porous thin films without damage of the underlying $\mathrm{SiO}_{2}$ layer and with a high coverage of O-terminated sites. The NCD surface topography, roughness, and coverage of the surface groups have been characterized by SEM, AFM and XPS methods. The EDIS sensors with O-terminated NCD film treated in oxidizing boiling mixture for $45 \mathrm{~min}$ show a $\mathrm{pH}$ sensitivity of about $50 \mathrm{mV} / \mathrm{pH}$. The $\mathrm{pH}$-sensitive properties of the NCD have been used to develop an EDIS-based penicillin biosensor with high sensitivity $(65-70 \mathrm{mV} / \mathrm{dec}$ ade in the concentration range of 0.25 $2.5 \mathrm{mM}$ penicillin $\mathrm{G})$ and low detection limit $(5 \mu \mathrm{M})$. The results of label-free electrical detection of layer-by-layer adsorption of charged polyelectrolytes are presented, too.
\end{abstract}

\section{INTRODUCTION}

Artificially grown diamond is a promising transducer material for chemical and biological sensing, as it is widely considered as biocompatible, displays outstanding electrical and electrochemical properties, and allows the direct coupling of biomolecules onto the diamond surface [1-5]. Among the various proposed concepts for the development of diamond-based chemical sensors and biosensors, the semiconductor field-effect platform is one of the most attractive approaches. Most of diamond-based field-effect (bio-)chemical sensors reported have been realized on a transistor structure by using hydrogen $(\mathrm{H})$-terminated polycrystalline or monocrystalline diamond films as an active transducer material [6-8].

Owing to the simplicity of the layout, the absence of a complicated encapsulation procedure and thus, an easier and cost-effective fabrication, capacitive field-effect structures are especially suited for (bio-)chemical sensor applications. Therefore, recently, we have introduced a capacitive field-effect electrolyte-diamond-insulator-semiconductor (EDIS) structure as a platform for (bio-)chemical sensing [9-11]. This work summarizes recent experimental results on the development of EDIS sensors for the detection of $\mathrm{pH}$, penicillin concentration and layer-bylayer adsorbed charged macromolecules using an oxygen (O)-terminated nanocrystalline diamond (NCD) films as transducer material. 


\section{EXPERIMENTAL}

Undoped NCD thin films of $\sim 100 \mathrm{~nm}$ thickness were grown on a p-Si-SiO $2(\rho=1-10 \Omega \mathrm{cm}$, $50 \mathrm{~nm}$ thermally grown $\left.\mathrm{SiO}_{2}\right)$ structure by means of microwave $(2.45 \mathrm{GHz})$ plasma-enhanced chemical vapor deposition from a mixture of methane $\left(\mathrm{CH}_{4}\right)$ and hydrogen $\left(\mathrm{H}_{2}\right)$ in an ASTeX 6500 reactor. Prior to growth, the $\mathrm{SiO}_{2}$ surface was seeded with a monodisperse colloid of nanocrystalline diamond particles in water with an ultrasonic bath. The NCD growth process on a $\mathrm{SiO}_{2}$ as well as an additional surface treatment in oxidizing medium have been optimized to yield high $\mathrm{pH}$-sensitive non-porous O-terminated NCD films without damage of the underlying $\mathrm{SiO}_{2}$ layer. An $\mathrm{Al}$ film was deposited on the rear side of the Si chip as a contact layer. The chip size of the EDIS sensors has been $10 \times 10 \mathrm{~mm}^{2}$. For more details about the preparation of NCD films, see [12,13].

Typically, as prepared NCD surfaces are hydrogen $(\mathrm{H})$-terminated. In order to obtain $\mathrm{O}-$ terminated surfaces, the NCD films were treated in an oxidizing mixture of $\mathrm{H}_{2} \mathrm{SO}_{4}$ and $\mathrm{KNO}_{3}$. The NCD films have been physically characterized by means of scanning electron microscopy (SEM), atomic force microscopy (AFM) and X-ray photoelectron spectroscopy (XPS) methods. The SEM micrograph in Figure 1 exemplarily demonstrates the surface morphology of a $100 \mathrm{~nm}$ thick NCD film. The film comprised randomly orientated fine grains and was totally closed. No visible pores in the film or damages of the underlying $\mathrm{SiO}_{2}$ layer have been observed.

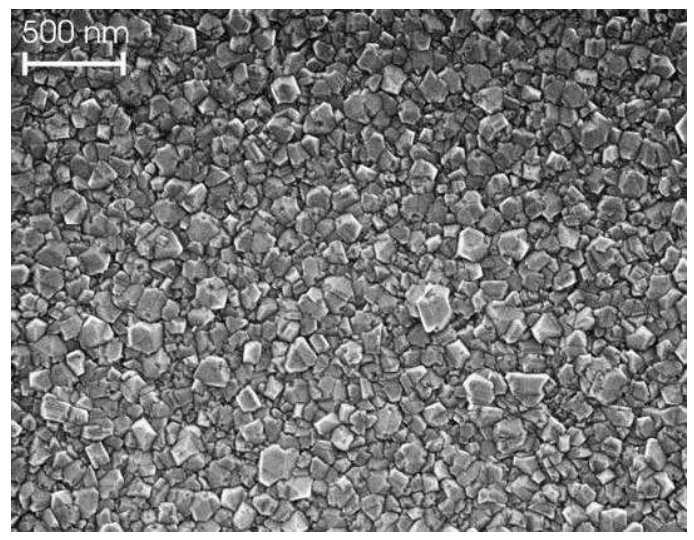

Figure 1. SEM picture of the surface morphology of a $100 \mathrm{~nm}$ thick NCD film grown on a p-Si$\mathrm{SiO}_{2}$ structure.

Additional AFM analysis of the diamond films was performed in intermittent contact mode (BioMat Workstation, JPK Instruments - Germany) and revealed a nanostructured surface consisting of NCD grains having a size of $\sim 100 \mathrm{~nm}$ with an average surface roughness of $11 \mathrm{~nm}$ as depicted in Figure 2.

The EDIS sensors have been electrochemically characterized in buffer solutions with different $\mathrm{pH}$ values by means of capacitance-voltage and constant-capacitance (ConCap) method using an impedance analyzer (Zahner Elektrik). For the experiments, the EDIS sensors were mounted into a home-made measuring cell, sealed by an O-ring and contacted on its front side by the electrolyte and on its rear side by a gold-plated pin. A conventional liquid-junction $\mathrm{Ag} / \mathrm{AgCl}$ electrode was used as a reference electrode. For more detailed information on the measurement set-up, see [9]. 

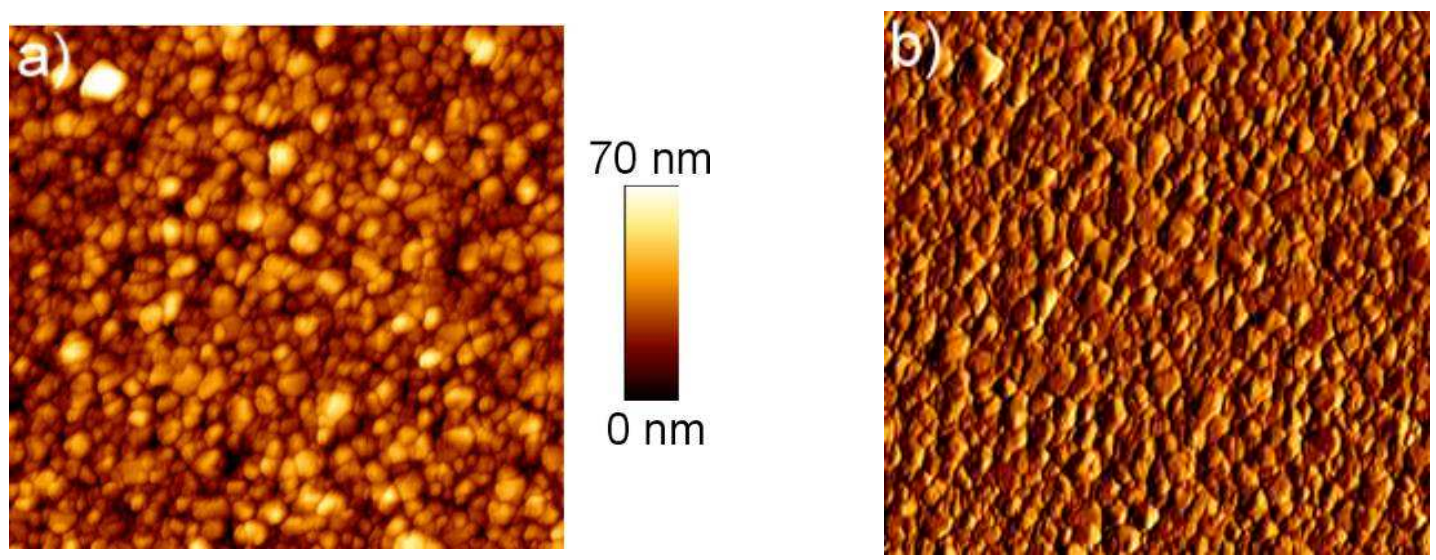

Figure 2. AFM height (a) and phase (b) images of a NCD film. The scan size is $2 \times 2 \mu \mathrm{m}^{2}$.

\section{RESULTS AND DISCUSSION}

\section{EDIS-based pH sensor}

The $\mathrm{pH}$ sensitivity of O-terminated EDIS sensors has been studied by means of ConCap method. Figure 3 demonstrates exemplarily a typical dynamic $\mathrm{pH}$-response (a) and calibration curve (b) of an EDIS structure with a $100 \mathrm{~nm}$ thick O-terminated NCD film oxidized by wet chemical oxidation for about $45 \mathrm{~min}$. The ConCap signal has been recorded in Titrisol buffer solutions with different $\mathrm{pH}$ values from $\mathrm{pH} 4$ to $\mathrm{pH}$ 12. The O-terminated EDIS sensors displayed a $\mathrm{pH}$ sensitivity of $48-50 \mathrm{mV} / \mathrm{pH}$. The $\mathrm{pH}$-sensitive properties of NCD films can be explained by the site-binding model, similar to ion-sensitive field-effect transistors. The surface potential of the diamond film could be influenced by the presence of hydroxyl groups at the oxidized diamond surface, resulting in the $\mathrm{pH}$-dependent modulation of the space-charge capacitance in the semiconductor and the sensor output signal.
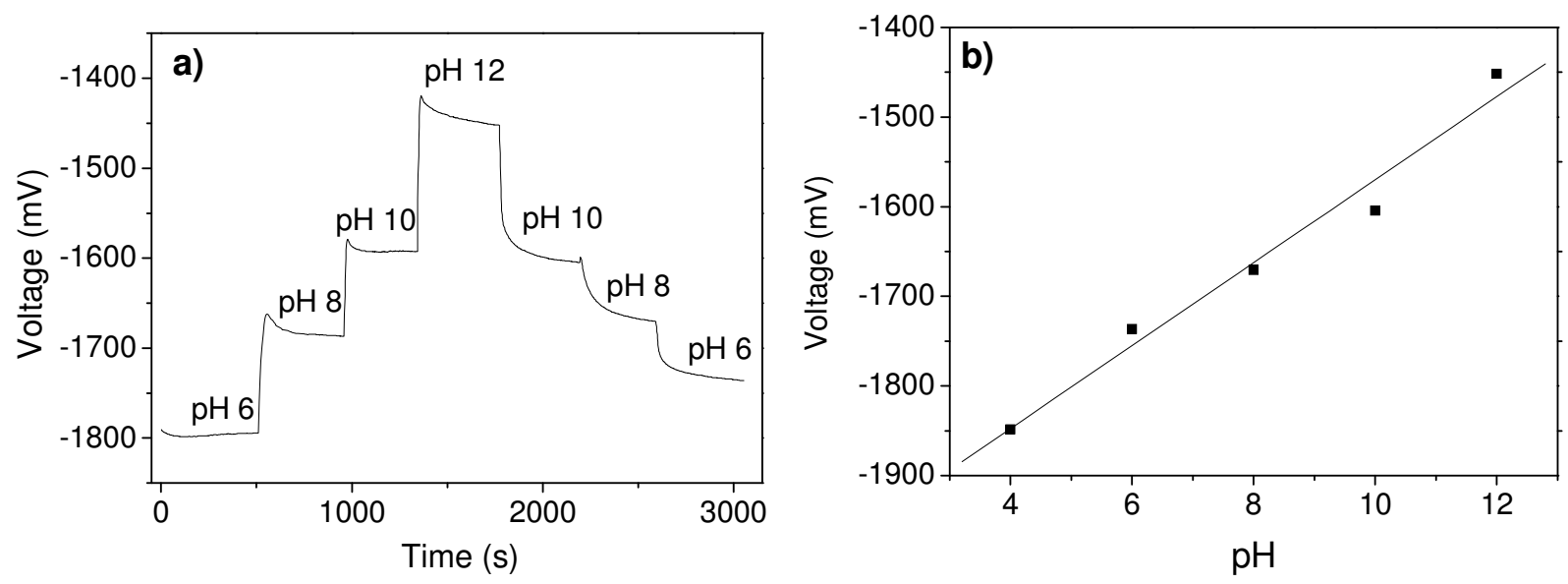

Figure 3. Typical ConCap response (a) and calibration curve (b) for O-terminated NCD film recorded in Titrisol buffer of different $\mathrm{pH}$ values from $\mathrm{pH} 6$ to $\mathrm{pH} 12$. 
The obtained values of $\mathrm{pH}$ sensitivity of NCD films are slightly lower than for $\mathrm{Al}_{2} \mathrm{O}_{3}$, $\mathrm{Si}_{3} \mathrm{~N}_{4}$, and $\mathrm{Ta}_{2} \mathrm{O}_{5}$ that have often been used as $\mathrm{pH}$-sensitive transducer materials for field-effect devices [14]. The main advantages of NCD films are their chemical inertness and biocompatibility as well as the possibility of interfacing to biological systems by direct coupling of biomolecules onto the diamond surface via carbon groups.

\section{EDIS-based penicillin biosensor}

The high $\mathrm{pH}$-sensitive properties of the EDIS structures have been used to develop a penicillin biosensor. The EDIS penicillin biosensor detects the local $\mathrm{pH}$ change near the surface as a result of the catalyzed hydrolysis of penicillin by the enzyme penicillinase. Figure 4 demonstrates an example of the dynamic ConCap response of the developed EDIS-based penicillin biosensor. In this experiment, the enzyme penicillinase (EC 3.5.2.6., Bacillus cereus from Sigma, specific activity: 1650 units/mg protein) was adsorptively immobilized directly onto the O-terminated NCD surface. The measurements have been performed in $0.5 \mathrm{mM}$ polymix

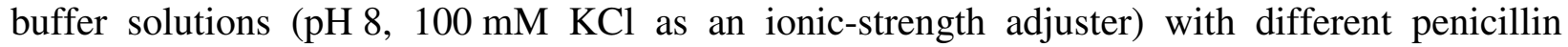
concentrations from $5 \mu \mathrm{M}$ to $2.5 \mathrm{mM}$. The penicillin solutions were prepared by dissolving penicillin $\mathrm{G}$ (benzyl penicillin, 1695 units/mg, Sigma) in the working buffer.

With increasing penicillin concentration, the concentration of the $\mathrm{H}^{+}$ions resulting from the penicillin hydrolysis is increased, too. As a result, the voltage that is necessary in order to adjust the constant capacitance raises. The freshly prepared NCD-based penicillin biosensor possessed a low detection limit of $5 \mu \mathrm{M}$ and a high sensitivity of $65-70 \mathrm{mV} / \mathrm{decade}$ in the concentration range from 0.25 to $2.5 \mathrm{mM}$ penicillin $\mathrm{G}$.

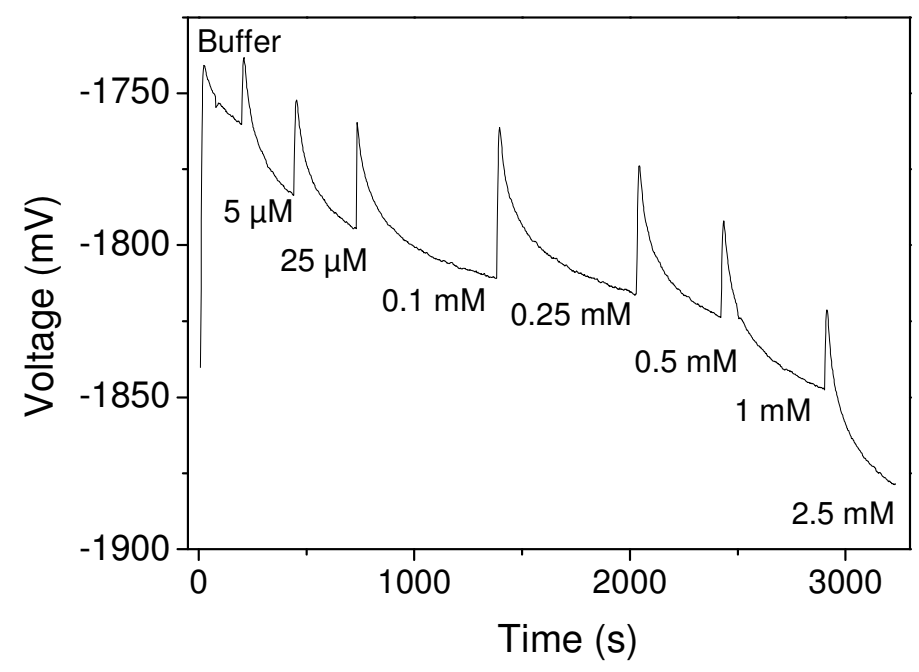

Figure 4. Typical ConCap response of the developed NCD-based penicillin biosensor.

\section{$\underline{\text { Label-free detection of charged macromolecules with EDIS sensor }}$}

In order to demonstrate functional capabilities of NCD films for multi-sensing applications, the realized EDIS structures with O-terminated NCD film have been used for a label-free electrical detection of charged macromolecules. The positively charged PAH (Poly 
(allylamine hydrochloride)) and negatively charged PSS (Poly (sodium 4-styrene sulfonate)) polyelectrolytes (PE) were chosen as model system. The multilayers of PAH/PSS were obtained by using the layer-by-layer assembly technique (see e.g., [15-17]) by sequential adsorption of PAH and PSS from the respective PE solution (50 $\mu \mathrm{M}$ PAH or PSS, $\mathrm{pH}$ 5.4). During the experiment, the EDIS sensors were consecutively exposed to the respective PE solution for a time necessary for the adsorption of each single monolayer, followed by rinsing in electrolyte solution. We started the formation of the PE multilayer onto O-terminated NCD with positively charged PAH.

Alternating potential changes, having the tendency to decrease with increasing the number of adsorbed polyelectrolyte layers (from 35-40 mV for first PE layers to 2-4 $\mathrm{mV}$ for an EDIS structure with 14-15 PE layers), have been observed after the adsorption of each PE layer. The adsorption of negatively charged PSS shifts the sensor signal towards the direction of a more negative surface charge, whereas the adsorption of the positively charged PAH shifts the sensor signal into the direction corresponding to a more positive charged NCD surface. Thus, the molecular layers induce an interfacial potential change resulting in alternating changes in the flat-band voltage, and therefore, in the output signal of the EDIS structure.

\section{CONCLUSIONS}

The presented results demonstrate the potential of field-effect capacitive EDIS structures having NCD films as transducer material for multi-parameter sensing of different (bio-)chemical quantities. The immobilization of other biomolecules, like DNA, proteins, on NCD films for extending the biosensor capabilities will be subject of future research.

\section{ACKNOWLEDGMENTS}

The authors thank H. P. Bochem for technical support. Part of this work was supported by the School for Life Sciences of the Transnational University Limburg, the IWT-SBO project "CVD diamond: a novel multifunctional material for high temperature electronics, high power/high frequency electronics and bioelectronics"), the Ministerium für Innovation, Wissenschaft, Forschung und Technologie des Landes Nordrhein-Westfalen, and the Bundesministerium für Bildung und Forschung (Germany).

\section{REFERENCES}

1. J. Rubio-Retama, J. Hernando, B. López-Ruiz, A. Härtl, D. Steinmüller, M. Stutzmann, E. López-Cabarcos, and J. A. Garrido, Langmuir 22, 5837 (2006).

2. R. J. Hamers, J. E. Butler, T. Lasseter, B. M. Nichols, J. N. Russell Jr., K.-Y. Tse, and W. Yang, Diamond Relat. Mater. 14, 661 (2005).

3. C. E. Nebel, B. Rezek, D. Shin, H. Uetsuka, and N. Yang, J. Phys. D: Appl. Phys. 40, 6443 (2007).

4. V. Vermeeren, N. Bijnens, S. Wenmackers, M. Daenen, K. Haenen, O. A. Williams, M. Ameloot, M. van de Ven, P. Wagner, and M. Michiels, Langmuir 23, 13193 (2007).

5. S. Wenmackers, V. Vermeeren, M. van de Ven, M. Ameloot, N. Bijnens, K. Haenen, L. Michiels, and P. Wagner, Phys. Stat. Sol. (a) 206, 391 (2009). 
6. K.-S. Song, M. Degawa, Y. Nakamura, H. Kanazawa, H. Umezawa, and H. Kawarada, J. Appl. Phys. 43, L814 (2004).

7. K.-S. Song, G.-J. Zhang, Y. Nakamura, K. Furukawa, T. Hiraki, J.-H. Yang, T. Funatsu, I. Ohdomari, and H. Kawarada, Phys. Rev. E 74, 041919 (2006).

8. C. E. Nebel, D. Shin, B. Rezek, N. Tokuda, H. Uetsuka, H. Watanabe, H., J. $R$. Soc.Interface 4, 439 (2007).

9. M. H. Abouzar, A. Poghossian, A. Razavi, O. A. Williams, N. Bijnens, P. Wagner, and M. J. Schöning, Biosens. Bioelectron. 24, 1298 (2009).

10. A. Poghossian, M. H. Abouzar, A. Razavi, M. Bäcker, N. Bijnens, O. A. Williams, K. Haenen, W. Moritz, P. Wagner, and M. J. Schöning, Electrochim. Acta 54, 5981 (2009).

11. M. H. Abouzar, A. Poghossian, A. Razavi, A. Besmehn, N. Bijnens, O. A. Williams, K. Haenen, P. Wagner, and M. J. Schöning, Phys. Stat. Sol. (a) 205, 2141 (2008).

12. O. A. Williams, O. Douhéret, M. Daenen, K. Haenen, E. Osawa, and M. Takahashi, Chem. Phys. Lett. 445, 225 (2007).

13. M. Daenen, O. A. Williams, J. D'Haen, K. Haenen, and M. Nesladek, Phys. Stat. Sol. (a) 203, 3005 (2006).

14. A. Poghossian, and M. J. Schöning, "Silicon-based Chemical and Biological Field-Effect Sensors", Encyclopedia of Sensors, vol. 9, ed. C. A. Grimes, E. C. Dickey, and M. V. Pishko (American Scientific Publishers, 2006) pp.1-71.

15. G. Decher, M. Eckle, J. Schmitt, and B. Struth, Curr. Opin. Coll. Interface Sci. 3, 32 (1998).

16. M. Schönhoff, Curr. Opin. Coll. Interface Sci. 8, 86 (2003).

17. M. Schönhoff, V. Ball, A. R. Bausch, C. Dejugnat, N. Delorme, K. Glimel, R. v. Klitzing, and R. Steitz, Coll. Surf. A 303, 14 (2007). 